\title{
Comportement du technétium 99 et du molybdène stable dans un sol brun calcaire
}

\author{
A. SAAS, A. GRAUBY, J. L. DENARDI \\ et J. M. QUINAULT (*)
}

(Manuscrit reçu le 28 février 1979)

\begin{abstract}
RÉSUMÉ
Une expérimentation à l'aide d'un dépôt au sol de concentration variable en technétium 99 et constant en molybdate d'ammonium stable a permis de définir leur comportement physicochimique dans un sol brun calcaire. Cette étude a abouti à définir, d'une part, le lessivage du technétium et du molybdène dans le sol, d'autre part, l'accumulation superficielle en fonction du dépôt. Une formulation mathématique a pu être effectuée pour chacun de ces deux paramètres. En outre, il apparaît que la forme physico-chimique du technétium et du molybdène est conditionnée par la concentration du dépôt. Nous avons retenu pour la formulation mathématique les formes essentiellement disponibles pour le végétal (hydrosoluble et extractible au tampon de Crigg). L'apport de concentrations variables de technétium a permis d'évaluer la rétention maximale de cet isotope dans le sol brun calcaire utilisé; cette valeur s'élève à $0,12 \mu \mathrm{g} / \mathrm{g}$ de sol. Deux données essentielles se dégagent de cette étude : pour des apports faibles en technétium, celui-ci s'accumule à la surface du sol et est disponible pour les végétaux; pour des apports supérieurs à $0,01 \mu \mathrm{g} / \mathrm{g}$ de sol, le lessivage du technétium concerne une fraction importante du dépôt, il subsiste alors un excès de forme hydrosoluble donc assimilable par le végétal et disponible pour une lixiviation vers la nappe phréatique.
\end{abstract}

\begin{abstract}
An experiment was conducted using soil deposits of technetium 99 at varying concentrations and stable ammonium molybdate at constant concentrations. Their physico-chemical behaviour in a brown chalky soil seemed to depend on the deposit concentrations. Both technetium and molybdenum leaching in the soil and surface accumulation as a function of the deposits were defined. A mathematical formulation was derived for each parameter, considering the forms available to the plant (hydrosoluble and extractable with Crigg buffer).
\end{abstract}

(*) Commissariat à l'énergie atomique, I.P.S.N., Département de protection, Service d'études et recherches sur l'environnement, Centre d'études nucléaires de Cadarache, B.P. $\mathrm{n}^{\circ} 1,13115$ Saint-Paul-Lez-Durance.

RADIOPROTECTION, vOL. 14 - 0033-8451/1979/241/\$ 4.00/C Bordas-Dunod. 
By using deposits of technetium at varying concentrations, the maximum retention in a brown chalky soil was found to be $0.12 \mu \mathrm{g} / \mathrm{g}$ of soil. Two main data could be derived: for low deposits technetium was accumulated at the soil surface and available for the plants; for deposits above $0.01 \mu \mathrm{g} / \mathrm{g}$ of soil, technetium leaching interested a large fraction of the deposit, there remained then an excess of hydrosoluble form ready to be assimilated by plants and available for leaching toward underground waters.

La chaîne de fission du numéro atomique 99 conduit à obtenir essentiellement deux isotopes : le molybdène 99 et le technétium 99 selon le schéma ci-dessous; le rendement de la fission est de $6,2 \mathrm{p}$. cent, ce qui conduit à obtenir ces isotopes avec une certaine abondance. Par ailleurs, ces deux isotopes résultent également de l'activation $(n, \gamma)$ du molydène présent dans les structures du réacteur et celui produit par la chaîne 98 .

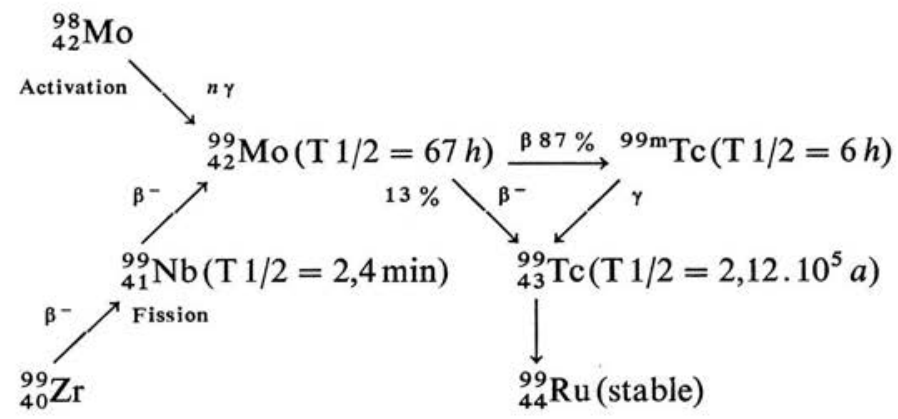

L'intérêt de ces isotopes découle également de deux autres considérations : le molybdène est un oligo-élément sur le plan agronomique, il intervient notamment dan: la fixation de l'azote par les légumineuses; le technétium appartient au groupe VII de la classification de Mendeléeff, tout comme le manganèse. Le technétium est chimiquement assez éloigné du manganèse en raison de la stabilité relativement plus grande des formes anioniques (pertechnétates); il se rapproche à cet égard du manganèse. Quoi qu'il en soit, les conditions d'oxydo-réduction du milieu récepteur sont des paramètres essentiels à la fois pour le technétium, le molybdène et le manganèse. Étant donné les similitudes des molybdates et des pertechnétates et la succession dans les chaînes de fission et d'activation notre étude s'intéresse à ces deux radionucléides.

Les travaux effectués récemment par LANDA et GAST [1, 2] font apparaître que le technétium 99 peut se fixer au niveau du sol; l'équilibre entre la solution et le sol est assez lent (une dizaine de jours); différentes expériences complémentaires ont permis de mettre en évidence le rôle de la matière organique et de l'activité microbienne sur la rétention de cet isotope par le sol. Par ailleurs, ces mêmes auteurs ont constaté une très grande disponibilité du technétium pour les végétaux. Partant de ces données notre expérimentation a un triple but :

- évaluer, d'une part, le lessivage du technétium et du molybdène dans un sol en fonction de la quantité de technétium déposée;

- évaluer, après une culture, la quantité de technétium résiduel en surface du sol et définir les formes physico-chimiques; 
- évaluer l'interaction du technétium et du molybdène au niveau du sol pour différentes valeurs du rapport $\mathrm{Mo} / \mathrm{Tc}$ du dépôt initial.

L'objectif final de cette expérimentation est d'établir une représentation mathématique de ces phénomènes afin de pouvoir calculer le dépôt au sol à partir des eaux d'irrigation, le lessivage vers la nappe phréatique et la disponibilité pour le végétal.

\section{MONTAGE EXPÉRIMENTAL ET CONDUITE DE L'EXPÉRIMENTATION}

Le sol utilisé pour cette expérimentation est un sol brun calcaire dont les principales caractéristiques physico-chimiques figurent au tableau I. Ce sol est communément répandu en France. Les réactions alcalines et oxydantes de ce sol favorisent par ailleurs l'assimilation du molybdène et probablement du technétium par le végétal. $\mathrm{Ce}$ sol renferme, en outre, une quantité non négligeable de manganèse dit échangeable $(4 \mathrm{mg} / 100 \mathrm{~g})$ et de manganèse dit actif $(8 \mathrm{mg} / 100 \mathrm{~g})$.

TABLEAU 1

CARACTÉRISTIQUES PHYSICO-CHIMIQUeS DU SOL BRUN CALCAIRE

\begin{tabular}{|c|c|c|c|c|c|c|c|}
\hline $\mathrm{pH}$ & $\begin{array}{c}\text { Matière } \\
\text { organique } \\
(\%)\end{array}$ & $\mathrm{C} / \mathrm{N}$ & $\begin{array}{c}\text { Argile } \\
(\%)\end{array}$ & $\begin{array}{c}\text { Capacité } \\
\text { d'échange } \\
(\mathrm{meq} / 100 \mathrm{~g})\end{array}$ & $\begin{array}{c}\text { Taux } \\
\text { de } \\
\text { saturation } \\
(\%)\end{array}$ & $\begin{array}{c}\text { Calcium } \\
\text { échangeable } \\
(\mathrm{meq} / 100 \mathrm{~g})\end{array}$ & $\begin{array}{c}\mathrm{CaCO}_{3} \\
(\%)\end{array}$ \\
\hline 7,80 & 1,05 & 8,5 & 18,0 & 8,9 & 100 & 29,1 & 28,5 \\
\hline
\end{tabular}

\begin{tabular}{|c|c|c|c|c|c|}
\hline $\begin{array}{c}\text { Silice } \\
\text { libre } \\
\%\end{array}$ & $\begin{array}{c}\text { Silice } \\
\text { hydrosoluble } \\
(\mathrm{mg} / 100 \mathrm{~g})\end{array}$ & $\begin{array}{c}\text { Fer } \\
\text { libre } \\
(\% \circ)\end{array}$ & $\begin{array}{c}\text { Mo } \\
\text { hydrosoluble } \\
(\mathrm{ppm})\end{array}$ & $\begin{array}{c}\text { Mo } \\
\text { assimilable } \\
(\mathrm{ppm})\end{array}$ & $\begin{array}{c}\text { Rapport } \\
\text { Mo } \frac{\text { hydrosoluble }}{\text { assimilable }}\end{array}$ \\
\hline 0,15 & 0,80 & 16,25 & 2,4 & 0,8 & 3,0 \\
\hline
\end{tabular}

Ce sol renferme donc initialement $2,4 \mathrm{mg} / \mathrm{kg}$ de molybdène soluble à l'eau (rapport d'extraction sol/eau $1 / 2$ ) et $0,8 \mathrm{mg} / \mathrm{kg}$ de molybdène assimilable extractible au tampon de Grigg (oxalate $\mathrm{NH}_{4} 24,9 \mathrm{~g} / \mathrm{l}$ + acide oxalique $12,6 \mathrm{~g} / \mathrm{l}$ à pH 3,3) après le traitement à l'eau. Sur le plan de l'absorption racinaire, ces deux formes sont assimilables par le végétal; cependant, le végétal s'intéresse par priorité aux formes hydrosolubles.

Par lot de $2 \mathrm{~kg}$ de sol on apporte une quantité constante de $20 \mu \mathrm{g} / \mathrm{g}$ de molybdène (molybdate de $\mathrm{NH}_{4}$ ) afin de lever toute carence éventuelle et d'amener le taux à un niveau tel que l'erreur analytique soit réduite au stricte minimum; une quantité croissante de technétium 99 (pertechnétate de $\mathrm{NH}_{4}$ ) selon les valeurs rassemblées dans le tableau II.

Chaque sol reçoit le molybdène et le technétium en une seule fois à la surface $(150 \mathrm{ml}$ d'eau d'irrigation) tout de suite après le repiquage des plants de salade. L'évolution physico-

vOL. $14-\mathrm{N}^{\circ} 4$ 
chimique se fait donc durant la période de la végétation et sous la dépendance des paramètres chimiques, biochimiques et microbiologiques $\mathrm{du}$ sol.

L'élution de ces éléments s'effectue par apport d'eau (eau de la Durance) à raison de six irrigations représentant environ $240 \mathrm{~mm}$ de hauteur d'eau (soit $120 \mathrm{~mm} / \mathrm{mois}$ d'évapotranspiration potentielle). Après la récolte de la culture de salade, on prélève l'horizon superficiel $(0-2 \mathrm{~cm})$ pour effectuer le dosage des formes de technétium et de molybdène résiduel. Les percolats de chaque sol ont été collectés au $5,12,21$, et $35^{\circ}$ jour après le dépôt.

TABLEAU II

APPORT DE TECHNÉTIUM AU SOL

\begin{tabular}{|c|c|c|r|}
\hline \multicolumn{3}{|c|}{ Technétium apporté (avec deux répétitions) } \\
\cline { 1 - 2 } $\mathrm{N}^{\circ}$ & $\begin{array}{c}\text { Quantité } \\
\text { totale } \\
\text { en (nCi) }\end{array}$ & $\begin{array}{c}\text { Quantité } \\
\text { dans le sol } \\
(\mu \mathrm{g} / \mathrm{g})\end{array}$ & $\begin{array}{r}\text { Rapport } \\
(\mathrm{Mo} / \mathrm{Tc})\end{array}$ \\
\cline { 1 - 1 } & & $1,275.10^{-3}$ & 18268 \\
$2 \ldots$ & 43,4 & $2,55.10^{-3}$ & 9138 \\
$3 \ldots$ & 86,8 & $1,275.10^{-2}$ & 1827 \\
$4 \ldots$ & 434 & $5,1.10^{-2}$ & 457 \\
$5 \ldots$ & 1736 & $1,02.10^{-1}$ & 228,6 \\
$6 \ldots$ & 3472 & $3,06.10^{-1}$ & 76,2 \\
$7 \ldots$ & $1,04.10^{4}$ & 0,51 & 45,7 \\
$8 \ldots$ & $1,74.10^{4}$ & 1,02 & 22,9 \\
$9 \ldots$ & $3,47.10^{4}$ & 1,53 & 15,2 \\
$10 \ldots$ & $5,21.10^{4}$ & 2,55 & 9,2 \\
\hline
\end{tabular}

L'élution en présence des végétaux permet d'obtenir une interprétation plus proche de la réalité et, par ailleurs, les prélèvements par le végétal sont susceptibles de soustraire au lessivage des formes chimiques très mobiles.

\section{2. ÉLUTION DU TECHNÉTIUM ET DU MOLYBDÈNE}

\subsection{RÉSULTATS EXPÉRIMENTAUX}

La figure 1 représente les courbes d'élution du technétium dans chaque lot expérimental en fonction du temps. Cette figure comporte deux références : la quantité de technétium apportée par une eau d'irrigation à la CMAP $\left(3 \cdot 10^{-4} \mathrm{Ci} / \mathrm{m}^{3}=0,0175 \mu \mathrm{g} / \mathrm{ml}\right)$ et l'activité correspondante du sol par gramme en estimant que le dépôt est uniforme sur $20 \mathrm{~g} / \mathrm{cm}^{2}$, soit 12 à $15 \mathrm{~cm}$ d'épaisseur. Pour un apport de $40 \mathrm{~mm}$ de hauteur d'eau soit $4 \mathrm{ml} / \mathrm{cm}^{2}$, correspondant à une irrigation unique normale, le dépôt en technétium est de $0,07 \mu \mathrm{g} / \mathrm{cm}^{2}$, soit $3,5 \cdot 10^{-3} \mu \mathrm{g} / \mathrm{g}$ de sol; pour un apport de $600 \mathrm{~mm}$ de hauteur d'eau soit $60 \mathrm{ml} / \mathrm{cm}^{2}$, correspondant à une irrigation annuelle, le dépôt en technétium est de $1,05 \mu \mathrm{g} / \mathrm{cm}^{2}$, soit $5,26 \cdot 10^{-2} \mu \mathrm{g} / \mathrm{g}$ de sol. Nos apports expérimentaux concordent donc parfaitement avec les quantités pouvant être déposées au sol par des irrigations avec une eau à la CMAP. 


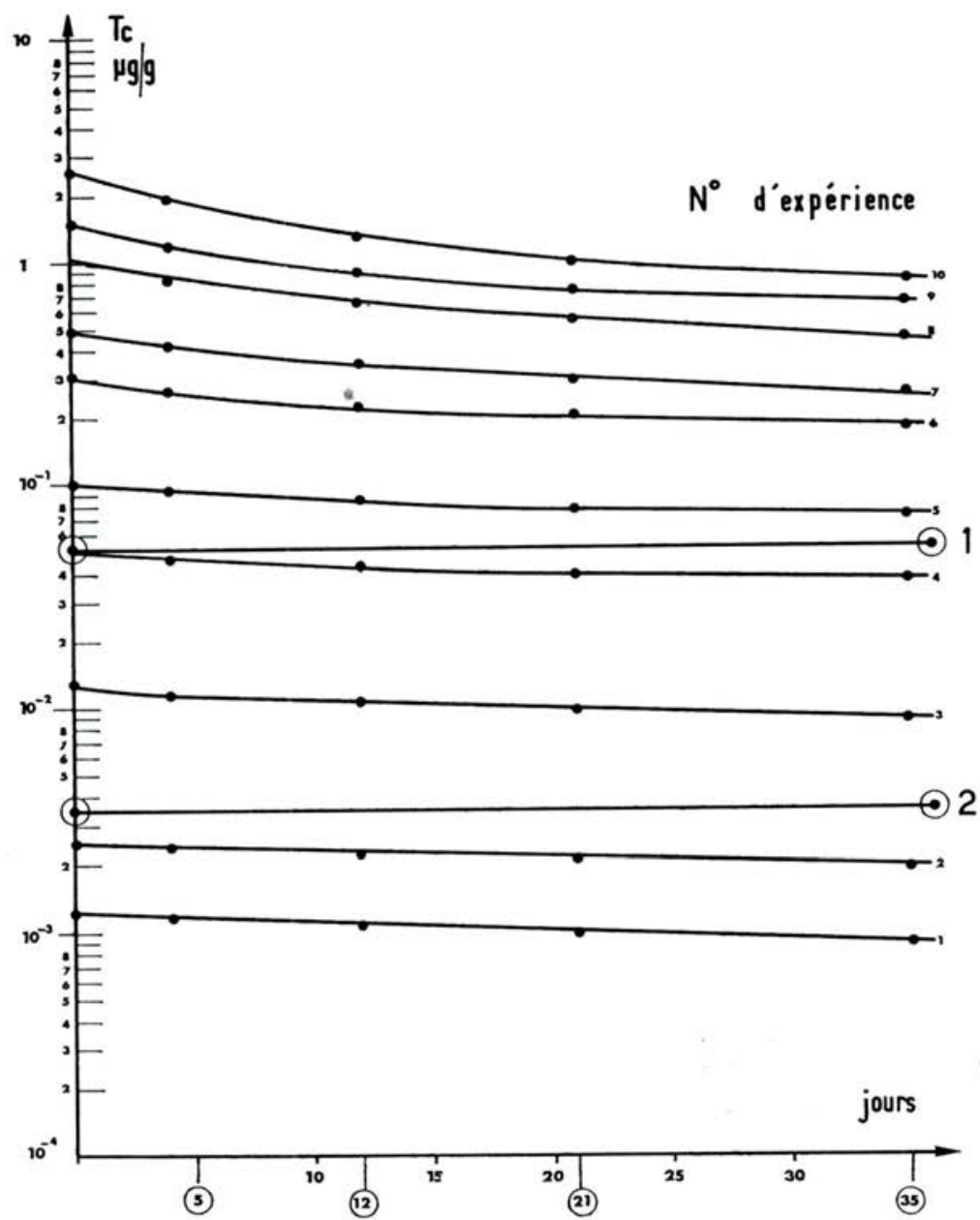

FIG. 1. - Technétium restant dans le sol en fonction du temps pour diverses concentrations initiales. 1 - apport $600 \mathrm{~mm} / \mathrm{an}$ à la CMAP : dépôt $\mu \mathrm{g} / \mathrm{g}$ à raison de $20 \mathrm{~g} / \mathrm{cm}^{2}$; 2 - apport $40 \mathrm{~mm} /$ irrigation à la CMAP : dépôt à raison de $20 \mathrm{~g} / \mathrm{cm}^{2}$.

Cette figure appelle les remarques suivantes : les pertes en technétium du sol sont faibles lorsque le dépôt est faible; par contre l'élution du technétium est importante lorsque le dépôt de technétium augmente. Ainsi les pertes en 1 mois se situent entre 20 et 25 p. cent pour une teneur en technétium du sol de $0,05 \mu \mathrm{g} / \mathrm{g}$ et aux environs de $60 \mathrm{p}$. cent pour un dépôt de $2,55 \mu \mathrm{g} / \mathrm{g}$. Il existerait donc une certaine proportionnalité de cette élution en fonction du dépôt initial du sol.

- l'élution se produit dans les premiers jours qui suivent le dépôt; ensuite le phénomène se ralentit considérablement; une fraction du technétium est donc retenue par le sol, l'autre

vOL. $14-\mathrm{N}^{\circ} 4$ 
fraction est lessivée parce que l'équilibre entre le sol et le technétium est lent, parce que le pertechnétate se fixe peu sur le sol. Si l'on se réfère aux expérimentations de GAST [2] l'évolution microbienne du pertechnétate est lente et seules les formes réduites sont retenues.

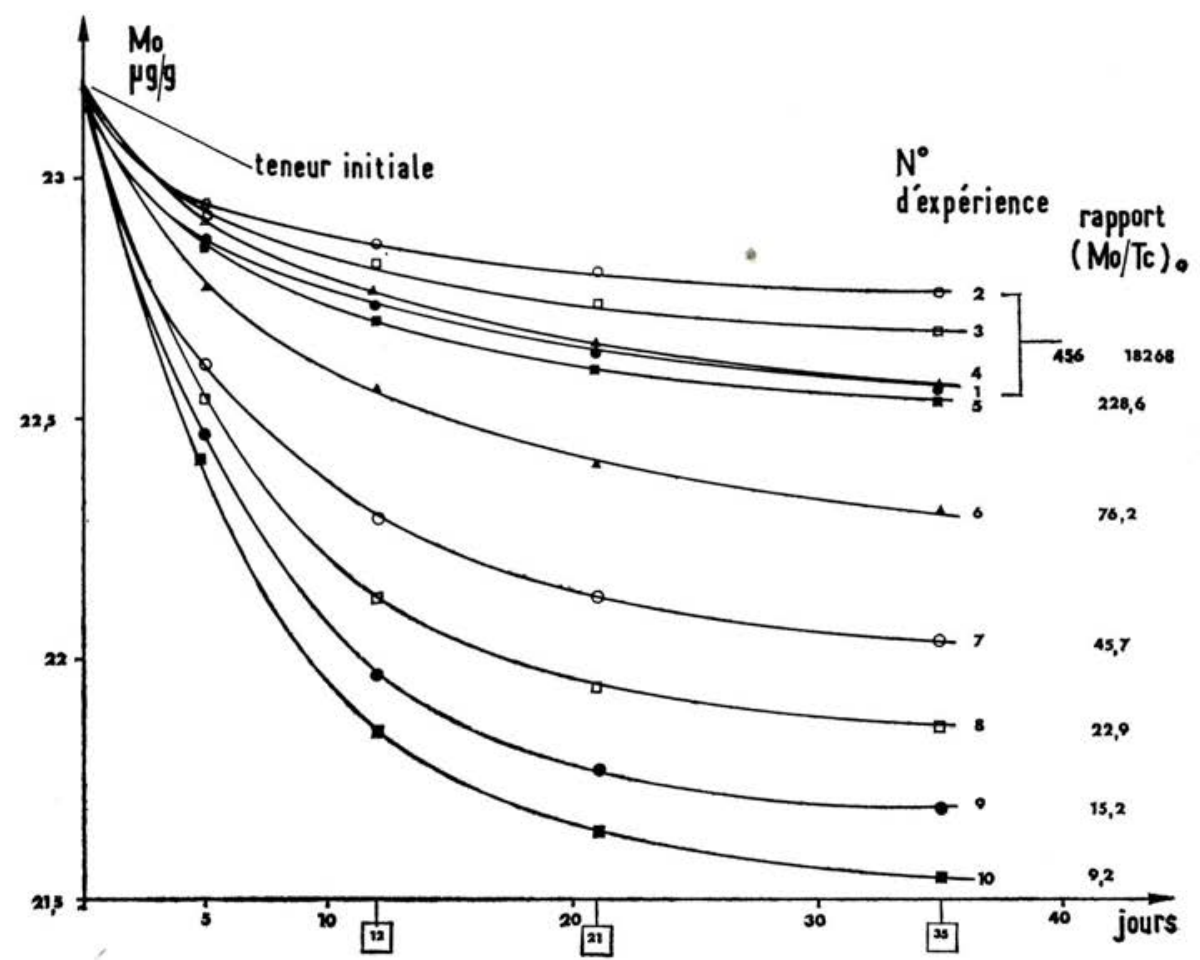

FIG 2. - Élution du molybdène en fonction du temps à partir d'un sol brun calcaire.

La figure 2 illustre 1'élution du molybdène en fonction du temps. On observe que la perte en molybdène est d'autant plus forte que la quantité de technétium apporté initialement est plus importante. Étant donné que la teneur en technétium d'un sol agricole est initialement infime $\left(10^{-13} \mathrm{ppm}\right)$, il paraît plus judicieux de travailler non pas en concentration mais avec le rapport $\mathrm{Mo} / \mathrm{Tc}$. On peut donc observer que la perte en molybdène est d'autant plus forte que le rapport Mo/Tc est faible. La cause de cette augmentation de l'élution est à attribuer certainement à la compétition, au niveau du sol, entre les ions molybdates et les ions pertechnétates. Ceci confirme par ailleurs que l'étude du cycle du technétium ne peut être séparée de celle du molybdène [3]. On peut, de ce fait, supputer que dans certains cas accidentels de dépôt sur des sols à faible teneur en molybdène, la fixation de technétium soit très élevée au niveau du sol et qu'il est alors vraisemblable qu'on ne puisse pas revenir naturellement en dessous du seuil de toxicité chimique compatible avec la croissance végétale $(1 \mu \mathrm{g} / \mathrm{g})$ [1]; ce seuil de toxicité varie, cependant, avec l'espèce végétale [6]. 
La figure 3, qui représente l'élution du molybdène en fonction du rapport $\mathrm{Mo} / \mathrm{Tc}$ à diverses dates, prend donc une signification précise en raison des observations précédentes et des répétitions. Les courbes d'élution sont sujettes à des changements de pente et l'on peu schématiquement différencier trois zones en fonction du rapport $\mathrm{Mo} / \mathrm{Tc}$ initial :

$$
\begin{aligned}
& \mathrm{Mo} / \mathrm{Tc}<40-50 ; \\
& 40-50<\mathrm{Mo} / \mathrm{Tc}<100 ; \\
& \mathrm{Mo} / \mathrm{Tc}>100
\end{aligned}
$$

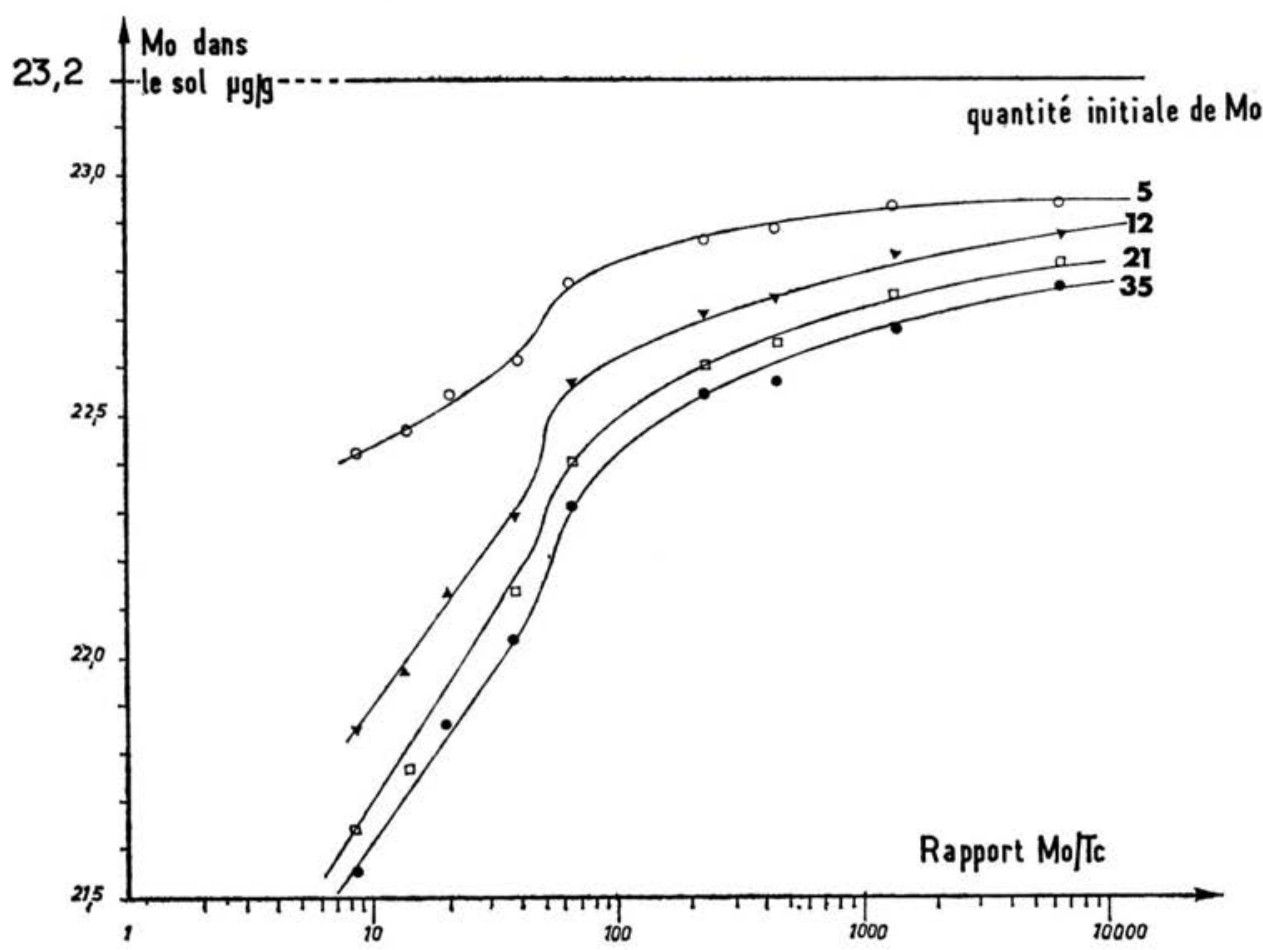

FIG. 3. - Quantité de molybdène restant dans le sol après 5, 12, 21, 35 jours en fonction du rapport initial $\mathrm{Mo} / \mathrm{Tc}$ pour un sol brun calcaire.

Cette observation fait ressortir que les pertes de technétium par lessivage sont à estimer, d'une part, en fonction du couple teneur en molybdène et technétium initialement présent dans le sol, d'autre part, en fonction des seuils définis ci-dessus pour le rapport $\mathrm{Mo} / \mathrm{Tc}$. Dans une des représentations mathématiques de l'élution que nous allons utiliser ultérieurement, nous nous fixerons deux seuils $\mathrm{Mo} / \mathrm{Tc}<40$ et $\mathrm{Mo} / \mathrm{Tc}>250$ car ce sont des valeurs au-delà desquelles nous pouvons assimiler les courbes précédentes à des droites.

La figure 4 montre clairement que le technétium remplace une fraction du molybdène dans le sol puisque la somme $\mathrm{Tc}+$ Mo tend vers une limite constante de l'ordre de $22,5 \mu \mathrm{g} / \mathrm{g}$ de sol lorsque le seuil Mo/Tc est supérieur à 100 et de l'ordre de $21,9 \mu \mathrm{g} / \mathrm{g}$ de sol lorsque ce seuil est inférieur à 40 . Dans cette somme limite, il y a, par ailleurs, d'autant

vol. $14-\mathrm{N}^{\circ} 4$ 


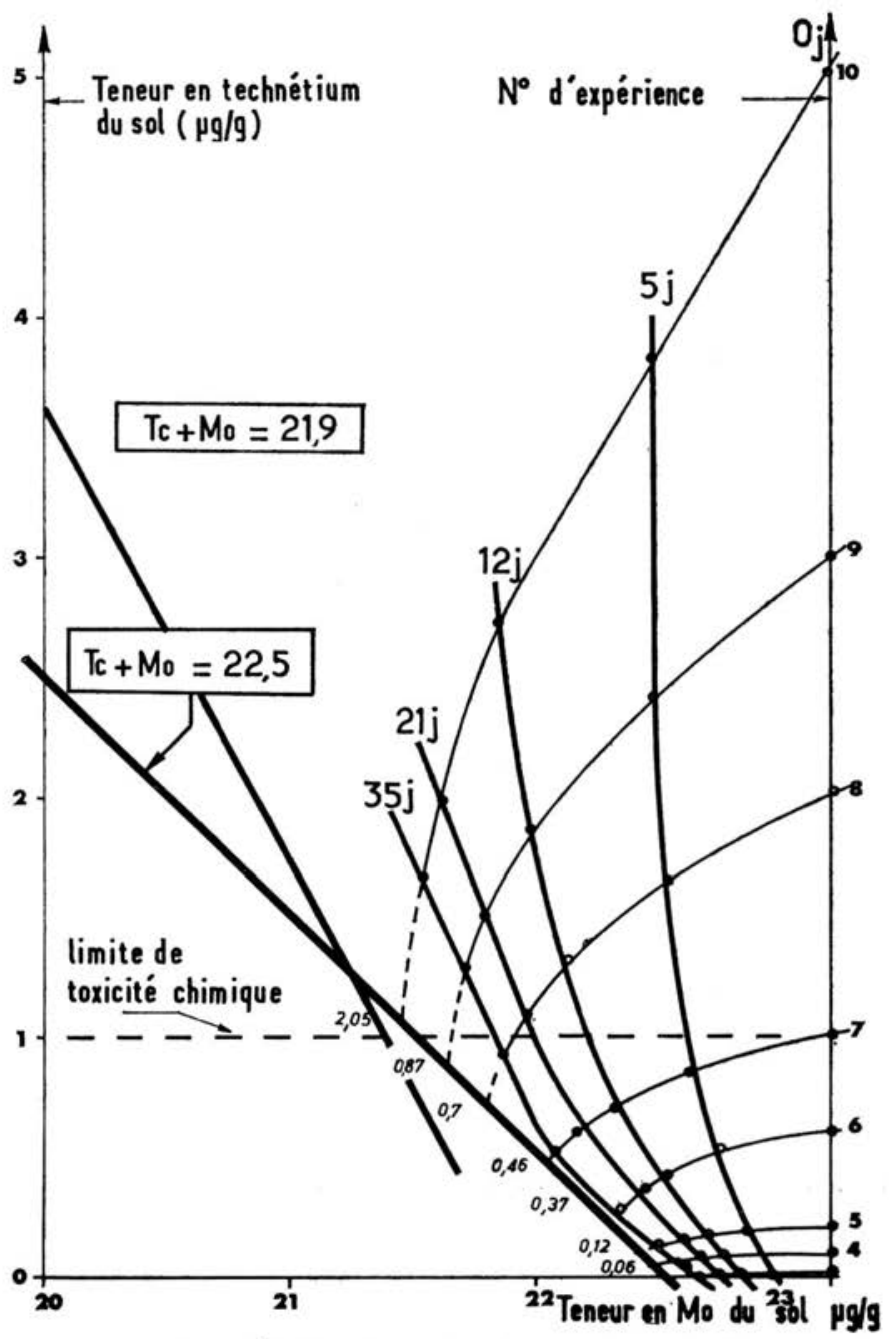

FIG. 4. - Élution du technétium et du molybdène à partir d'un sol brun calcaire en fonction du temps.

plus de technétium que le dépôt initial a été plus important. Les valeurs limites s'abaissent lorsque $\mathrm{Mo} / \mathrm{Tc}$ diminue à cause de la fixation très lente du technétium lorsqu'il est apporté en forte concentration; par ailleurs, l'assimilation du technétium par le végétal est importante pour les dépôts élevés. Ceci explique que nous ayons deux droites de pente différente. Deux conclusions essentielles sur le plan sanitaire se dégagent de l'examen de cette figure :

- $\mathrm{Si}$, dans un sol brun calcaire ayant des caractéristiques proches de celui qui a servi de support à notre expérimentation, on est en présence d'une teneur Mo + Tc supérieure 
à $22,5 \mu \mathrm{g} / \mathrm{g}$ ou $21,9 \mu \mathrm{g} / \mathrm{g}$ selon le rapport $\mathrm{Mo} / \mathrm{Tc}$ initial, l'élution ramènera la teneur d'origine à ces valeurs moyennes et ce d'autant plus lentement que la teneur en technétium est plus importante initialement.

- La teneur résiduelle en technétium après élution est d'autant plus élevée que le dépôt a été quantitativement plus fort. Cette teneur résiduelle peut d'ailleurs dépasser le seuil de toxicité $(1 \mu \mathrm{g} / \mathrm{g})$ tolérable pour le végétal évalué par GAST [1] soit durant un certain temps soit définitivement si l'on n'intervient pas artificiellement pour abaisser le seuil. Cette situation est probable en cas d'accident par exemple. Dans ce cas, il sera nécessaire de déplacer le technétium par des apports de molybdate.

Étant donné l'importance sur le plan sanitaire de ces observations, nous allons essayer à présent de formuler mathématiquement ces phénomènes.

\subsection{Modélisation mathématique de L'Élution}

Nous avons cherché à modéliser mathématiquement l'ensemble de ces résultats expérimentaux afin de pouvoir évaluer dans tous les cas de figure l'élution du technétium et du molybdène. Nous avons donc recherché une fonction qui représente l'élution d'un des éléments en fonction du temps et du rapport Mo/Tc initial :

$$
\text { Mo ou Tc élué }=F\left(t,(\mathrm{Mo} / \mathrm{Tc})_{0}\right) \text {. }
$$

Pour cela, on peut penser à représenter l'élution du molybdène ou du technétium par une somme de deux exponentielles : la première représentant schématiquement l'élution propre de l'élément et la seconde l'élution induite par l'adjonction de l'autre élément. Dans ce cas, la formulation de cette élution est la suivante :

$$
\text { Mo ou Tc élué }=A_{1}\left(1-e^{-\lambda_{1} t}\right)+A_{2}\left(1-e^{-\lambda_{2} t}\right) \text {. }
$$

Nous avons ensuite évalué les paramètres $A_{1}, A_{2}, \lambda_{1}, \lambda_{2}$ selon une méthode statistique (méthode de $\chi^{2}$ avec données concernant les formes de fonction exponentielles). A l'aide de valeurs ajustées, on minimise ensuite les écarts afin que les résultats calculés à l'aide de la formule précédente soient le plus proches possible des résultats expérimentaux.

Estimations des paramètres $\mathrm{A}_{1}, \mathrm{~A}_{2} \lambda_{1} \lambda_{2}$

\section{Molybdène}

$$
\begin{aligned}
& \lambda_{1}: 0,075(\mathrm{Mo} / \mathrm{Tc})_{0}^{0.3} \text { pour }(\mathrm{Mo} / \mathrm{Tc})_{0}<250 \text {, } \\
& 0,39 \text { pour }(\mathrm{Mo} / \mathrm{Tc})_{0}>250 \text {; }
\end{aligned}
$$

$\lambda_{2}: 0,03$ quel que soit le rapport $(\mathrm{Mo} / \mathrm{Tc})_{0}$;

$\mathrm{A}_{1}: 4(\mathrm{Mo} / \mathrm{Tc})_{0}^{0,5}$ pour $(\mathrm{Mo} / \mathrm{Tc})_{0}<250$, $21,9 \quad$ pour $(\mathrm{Mo} / \mathrm{Tc})_{0}>250$;

$\mathrm{A}_{2}: 0,08(\mathrm{Mo} / \mathrm{Tc})_{0}^{0,5}$ pour $(\mathrm{Mo} / \mathrm{Tc})_{0}<40$,

$$
0,5 \quad \text { pour }(\mathrm{Mo} / \mathrm{Tc})_{0} \geqslant 40 \text {. }
$$

On s'aperçoit donc que, conformément aux observations de la figure 3, l'élution du molybdène comporte plusieurs seuils qui sont déterminés par le rapport $(\mathrm{Mo} / \mathrm{Tc})_{0}$.

Lorsque le rapport $(\mathrm{Mo} / \mathrm{Tc})_{0}$ est supérieur à 250, l'élution maximale du molybdène est égale à $\mathrm{A}_{2}+\mathrm{A}_{1}$

$$
\left(\frac{\mathrm{Mo}}{\mathrm{Tc}}\right)_{0}>250 \quad \mathrm{Mo}_{\max }=0,5+21,9 \simeq 0,72 \mu \mathrm{g} / \mathrm{g} .
$$


Dans les autres cas, l'élution maximale s'écrit :

$$
\mathrm{Mo}_{\max }=\frac{4}{\sqrt{\mathrm{Mo} / \mathrm{Tc}}}(1+0,02 \mathrm{Mo} / \mathrm{Tc}) .
$$

\section{Technétium}

$\lambda_{1}: 0,15$;

$\lambda_{2}: 0,05$;

$\mathrm{A}_{1}: 12,6(\mathrm{Mo} / \mathrm{Tc})_{0}^{-3 / 2}$;

$\mathrm{A}_{2}: 10(\mathrm{Tc} / \mathrm{Mo})_{0}$.

L'élution du technétium s'écrit donc :

$$
\mathrm{Tc}_{\text {élué }}=12,6(\mathrm{Tc} / \mathrm{Mo})^{3 / 2}\left(1-\mathrm{e}^{-0,15 t}\right)+10 \mathrm{Tc} / \mathrm{Mo}\left(1-\mathrm{e}^{-0,05 t}\right) .
$$

Le maximum de technétium élué devient alors :

$$
\mathrm{Tc}_{\infty}=10 \frac{\mathrm{Tc}}{\mathrm{Mo}}(1+1,26 \sqrt{\mathrm{Tc} / \mathrm{Mo}})
$$

\section{Simplification de la formulation}

Cette formulation ne nous a pas entièrement satisfait sur le plan pratique. En effet, les coefficients des formules précédentes se calculent, pour le molybdène du moins, par des fonctions différentes valables seulement pour des seuils caractéristiques du rapport $(\mathrm{Mo} / \mathrm{Tc})_{0}$. Nous avons donc recherché une formulation plus commode en n'utilisant qu'un seul seuil pour Mo/Tc [formule (1), fig. 5] soit en faisant abstraction de la notion de seuil [formule 2, fig. 6]. Toujours dans le même esprit de simplification de la formulation, aux dépens d'ailleurs de la précision, nous avons retenu un paramètre intermédiaire unique $: x=(\mathrm{Tc} / \mathrm{Mo})_{0}^{0,25}$ avec lequel la représentation mathématique des résultats expérimentaux a été effectuée sur les figures 5 et 6 .

Élution du molybdène avec la formule (1) (fig. 5)

- seuil $=(\mathrm{Mo} / \mathrm{Tc})_{0} \leqslant 300$ :

$$
\text { Mo }=0,2\left(1-\mathrm{e}^{-0,4 t}\right)+0,5\left(1-\mathrm{e}^{-0,04 t}\right)
$$

- seuil $=(\mathrm{Mo} / \mathrm{Tc})_{0} \geqslant 300$ :

$$
\text { Mo }=3,5 x^{2}\left(1-\mathrm{e}^{-\frac{t}{10 x}}\right)+\left(2,9 x-3.5 x^{2}\right)\left(1-\mathrm{e}^{-0,04 t}\right)
$$

Élution du molybdène avec la formule (2) (fig. 6)

$$
\text { Mo }=0,2\left(1-\mathrm{e}^{-0,4 t}\right)+5 x^{3}\left(1-\mathrm{e}^{-0,1 t}\right)+\left(0,5-2 x^{3}\right)\left(1-\mathrm{e}^{-0,04 t}\right) .
$$




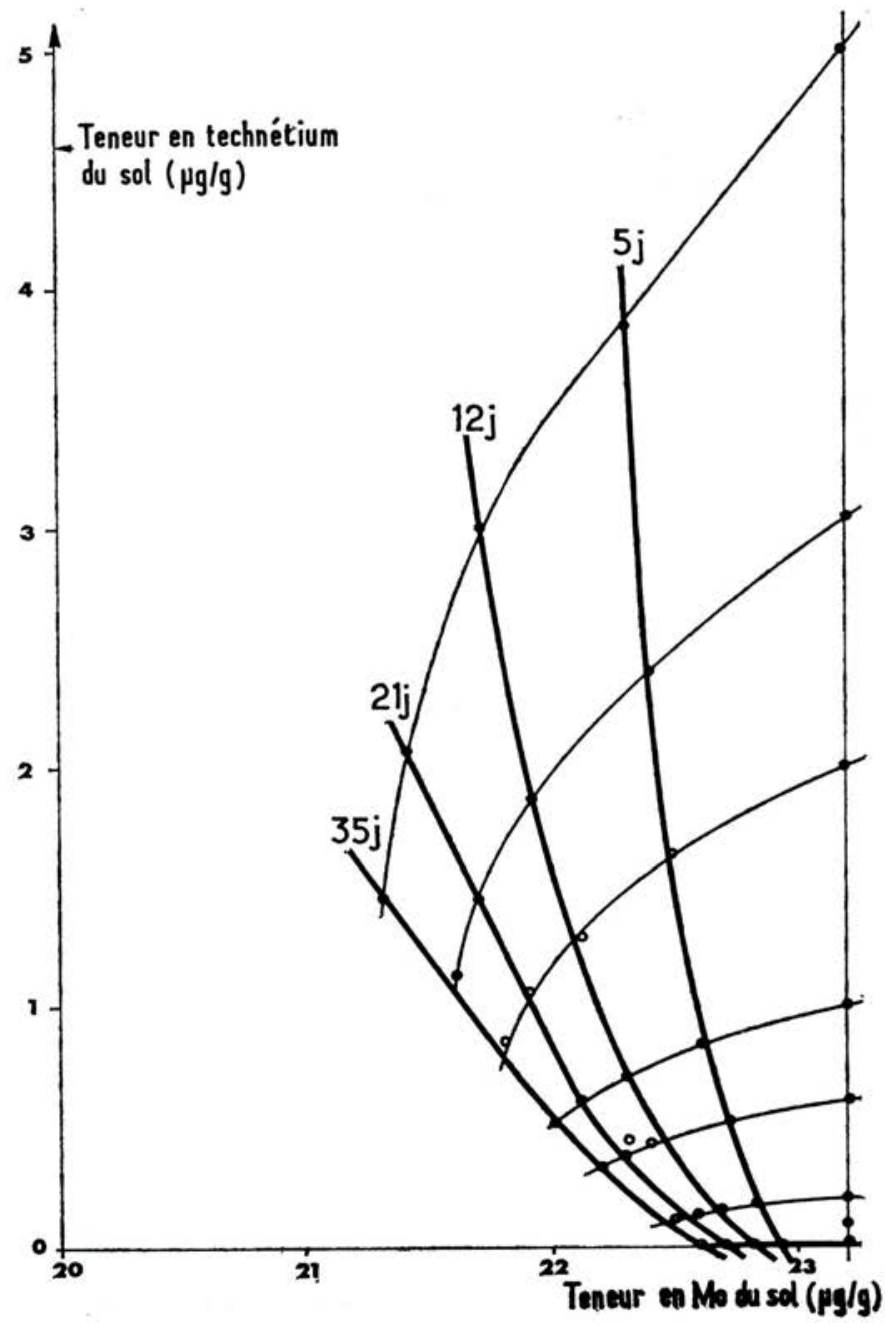

FIG. 5. - Élution du technétium et du molybdène à partir d'un sol brun calcaire en fonction du temps.

Avec ces formules, il est possible d'évaluer le maximum de molybdène élué : pour $(\mathrm{Mo} / \mathrm{Tc})_{0} \geqslant 300$ :

$$
\mathrm{Mo}_{\infty}=2,9 x
$$

pour $(\mathrm{Mo} / \mathrm{Tc})_{0}<300$ :

$$
\mathrm{Mo}_{\infty} \simeq 0,7 \text { à } 0,75 \mu \mathrm{g} / \mathrm{g} \text {. }
$$




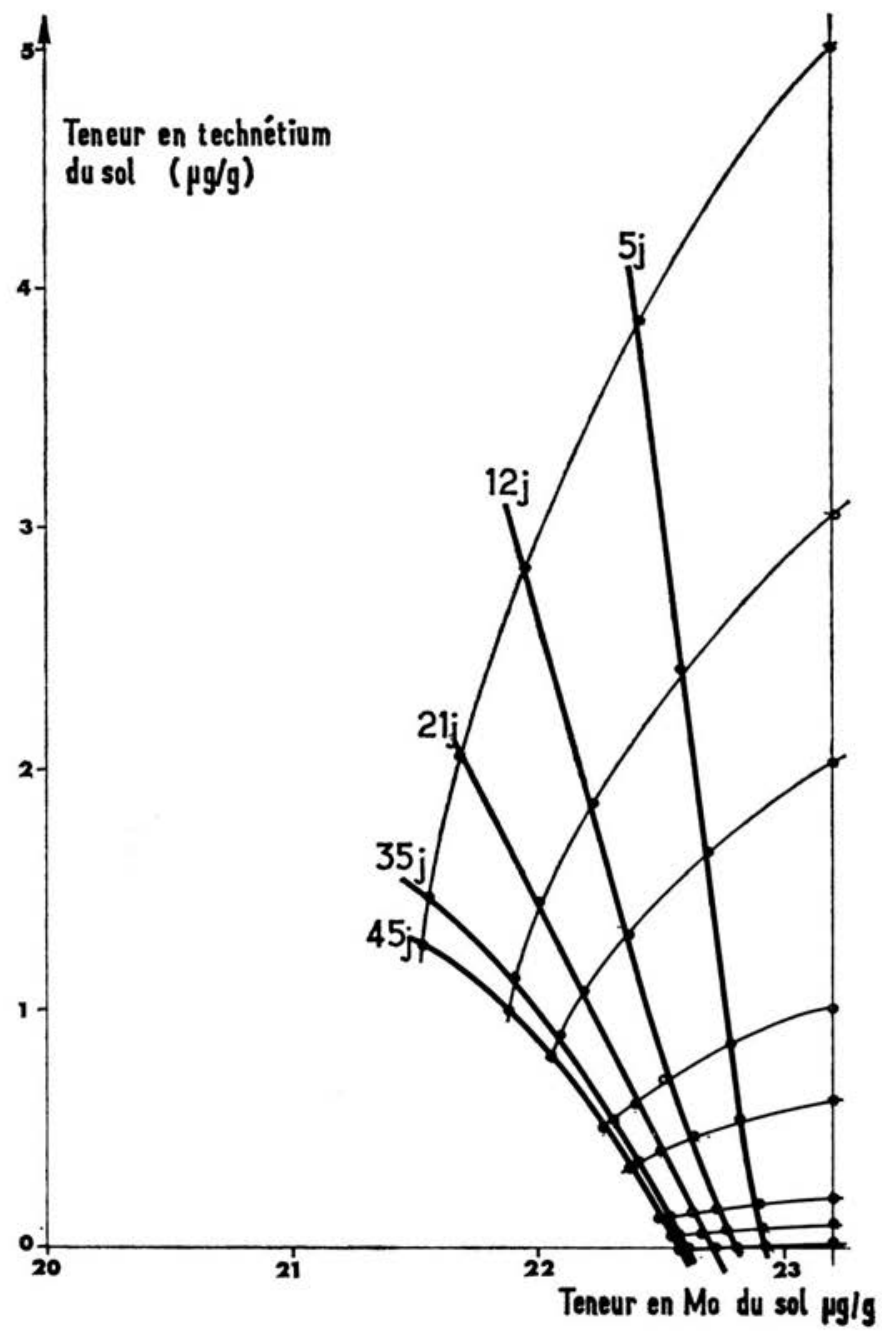

FIG. 6. - Élution du technétium et du molybdène à partir d'un sol brun calcaire en fonction du temps.

\section{Élution du technétium}

L'élution du technétium avec le paramètre intermédiaire $x=(\mathrm{Tc} / \mathrm{Mo})_{0}^{0,25}$ s'écrit comme suit :

$$
\mathrm{Tc}_{\text {élué }}=14 x^{5}\left[\left(1-\mathrm{e}^{-0,1 t}\right)+\left(1-\mathrm{e}^{-0,04 t}\right)\right] \text {. }
$$

Cette formule a été utilisée dans les représentations graphiques des figures 5 et 6 . 
Le maximum de technétium élué :

$$
\mathrm{Tc}_{\infty}=28 x^{5} .
$$

Cette dernière expression est très importante sur le plan sanitaire, car elle confirme que pour de très faibles quantités de technétium déposées au sol, c'est-à-dire dans des cas où le rapport $(T c / M o)_{0}$ est très petit, l'élution du technétium est quasiment nulle et la quantité déposée reste à la surface du sol; dans ces conditions il y a accumulation du technétium dans le sol. Ces conditions peuvent être obtenues lors d'irrigations avec des eaux à la CMAP ou inférieures à la CMAP. A la lumière de ces renseignements, il est donc normal d'évaluer l'accumulation à la surface du sol lors d'un dépôt par irrigation. Cependant, ayant calculé l'élution du technétium et du molybdène dans le sol, il paraît intéressant également de rechercher une formulation pour la quantité restante dans le sol lorsque le rapport Mo/Tc est élevé; à l'équilibre dans le sol brun calcaire, celle-ci s'écrit :

$$
(\mathrm{Tc}+\mathrm{Mo})_{\infty}=22,5-\left(14 x^{5}+3 x^{3}\right) \text {. }
$$

\section{COMPORTEMENT DU TECHNÉTIUM A LA SURFACE DU SOL}

L'étude de l'élution du technétium pour diverses concentrations déposées sur le sol nous a montré que cet isotope était retenu dans le cas où l'on apporte de faibles quantités. Il reste donc à connaître s'il y a accumulation à la surface du sol et dans cette hypothèse quelle sera la rétention maximale; autrement dit, à partir de quelle quantité déposée au sol y a-t-il, à proprement parler, un lessivage vers la nappe phréatique?

Dans le cas de notre expérimentation, l'apport de technétium et de molybdène a été réalisé en une seule fois lors de la plantation de salade. Afin de globaliser, l'élution par lessivage, le prélèvement du végétal par absorption racinaire, ainsi que les pertes par volatilisation, les prélèvements de la surface du sol $(0-2 \mathrm{~cm})$ ont été effectués après la récolte de salade.

Dans ces conditions, ces isotopes ont pu migrer en profondeur et, par ailleurs, l'incorporation par voie microbienne a pu s'effectuer durant une période suffisamment longue pour que ces éléments aient épousé des formes physico-chimiques telles qu'elles sont représentatives de l'équilibre. Chaque échantillon de sol a subi deux extractions successives : la première avec de l'eau pour déterminer la forme hydrosoluble, la seconde avec le tampon de Grigg pour évaluer la forme dite assimilable, étant entendu que ces deux formes sont disponibles pour le végétal.

\subsection{TeChnétium total EN SURface DU SOL}

Afin d'établir une relation entre l'accumulation à la surface du sol en fonction de la quantité initialement déposée, nous avons supposé que cet apport initial était uniformément réparti dans l'ensemble du sol. La figure 7 rassemble donc les valeurs expérimentales obtenues 2 mois après l'apport. L'examen de ces résultats nous montre qu'il y a effectivement une accumu-

VOL. $14-\mathrm{N}^{\circ} 4$ 
lation du technétium à la surface du sol même après élution et prélèvement par le végétal; cette accumulation est d'autant plus importante que la quantité déposée est plus faible. Le technétium se comporte donc comme un élément

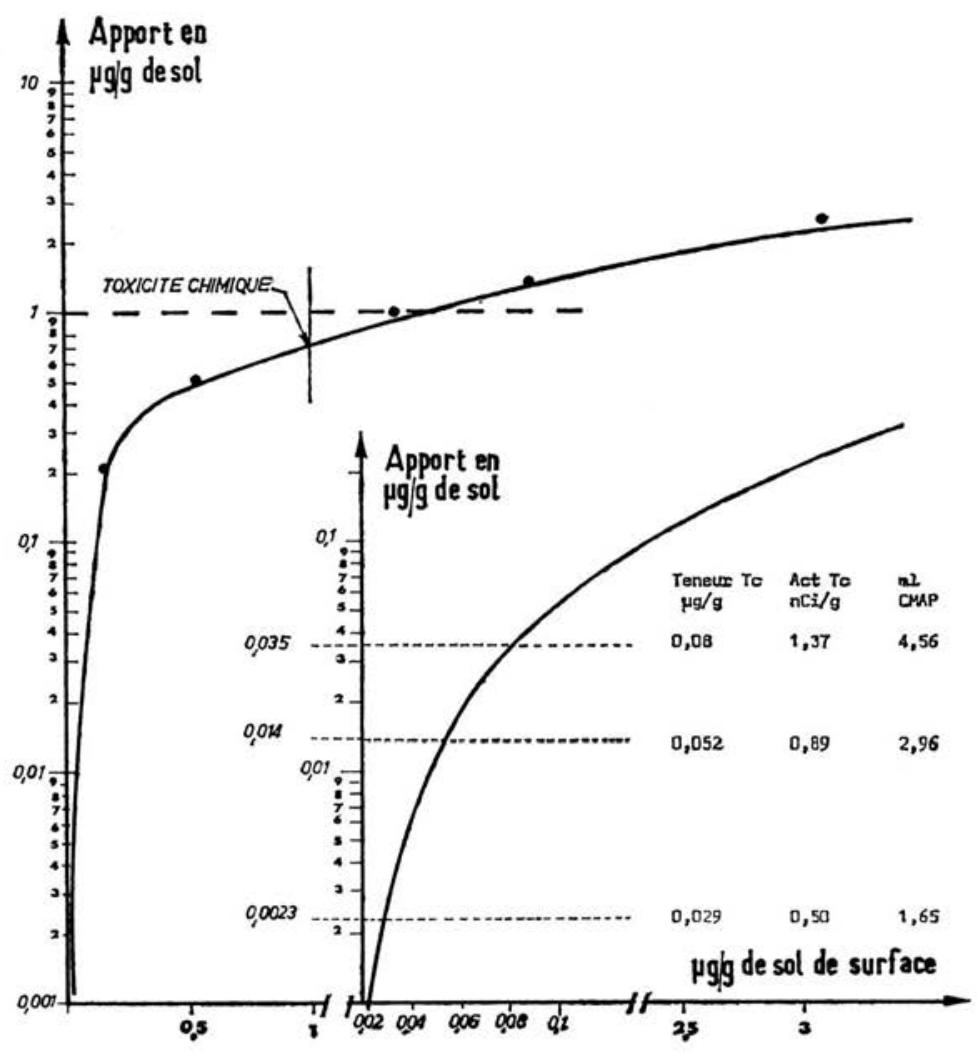

FIG. 7. - Technétium total en surface du sol en fonction de l'apport initial après une culture de salade.

stable et il y a donc un seuil de rétention maximal. De ce fait, il est possible de définir, d'une part, quel est le dépôt nécessaire pour atteindre le seuil de toxicité chimique et, d'autre part, si ce seuil peut être effectivement atteint dans ce sol brun calcaire.

Pour un apport équivalent au seuil de la toxicité chimique pour les racines de salade $(1 \mu \mathrm{g} / \mathrm{g}$ de sol), la teneur en technétium résiduel à la surface du sol est de $1,46 \mu \mathrm{g} / \mathrm{g}$. La valeur résiduelle, à la surface du sol correspondante, à $1 \mu \mathrm{g} / \mathrm{g}$ de sol est obtenue pour un apport de $0,71 \mu \mathrm{g} / \mathrm{g}$ qui équivaut à un dépôt de $21,3 \mu \mathrm{g} / \mathrm{cm}^{2}$ soit en activité $363,2 \mathrm{nCi} / \mathrm{cm}^{2}$ ou exprimée en hauteur d'eau d'irrigation à la CMAP : $12000 \mathrm{~mm}$ ou 20 ans d'irrigation à raison de $600 \mathrm{~mm} / \mathrm{an}$. Le seuil de toxicité chimique peut donc être atteint à la surface 
du sol dans un délai raisonnable avec des eaux d'irrigation à la CMAP. Le sol brun calcaire peut-il vraiment retenir une quantité aussi importante? Nous essaierons de répondre à cette question dans un paragraphe ultérieur.

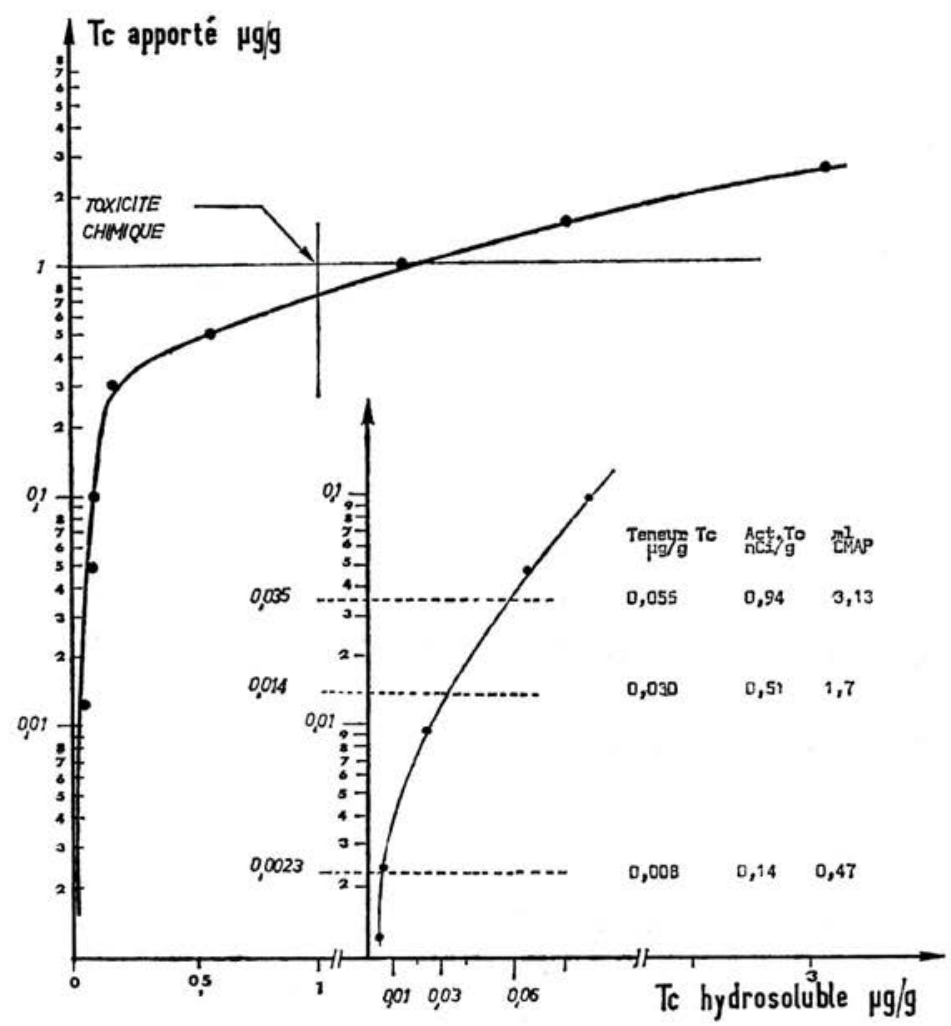

Fig. 8. - Technétium hydrosoluble dans la partie superficielle du sol après une culture de salade.

La courbe expérimentale de la figure 7 nous conduit, cependant, à évaluer l'accumulation en surface du sol à court terme $(1$ an) et pour des faibles quantités de dépôt. Pour un apport expérimental de $1,28 \cdot 10^{-3} \mu \mathrm{g} / \mathrm{g}$ de sol, la teneur en technétium de la surface du sol est voisine de $0,022 \mu \mathrm{g} / \mathrm{g}$. Il est donc possible à partir de cette courbe expérimentale d'évaluer la concentration pour des irrigations avec une eau à la CMAP au cours de la première année.

Irrigation unique de $40 \mathrm{~mm}$ de hauteur d'eau

Le dépôt est de $4 \mathrm{ml} / \mathrm{cm}^{2}$ soit $0,07 \mu \mathrm{g}$ de technétium; en supposant le dépôt uniforme sur $20 \mathrm{~cm}\left(30 \mathrm{~g}\right.$ de $\left.\mathrm{sol} / \mathrm{cm}^{2}\right)$, la teneur moyenne est de 
$0,0023 \mu \mathrm{g} / \mathrm{g}$. La teneur à la surface du sol d'après la courbe expérimentale de la figure 7 est de $0,029 \mu \mathrm{g} / \mathrm{g}$ soit $0,5 \mathrm{nCi} / \mathrm{g}$, cette activité correspond à $1,65 \mathrm{ml}$ d'eau à la CMAP. Le facteur de concentration après prélèvement par absorption racinaire est de 12,6.

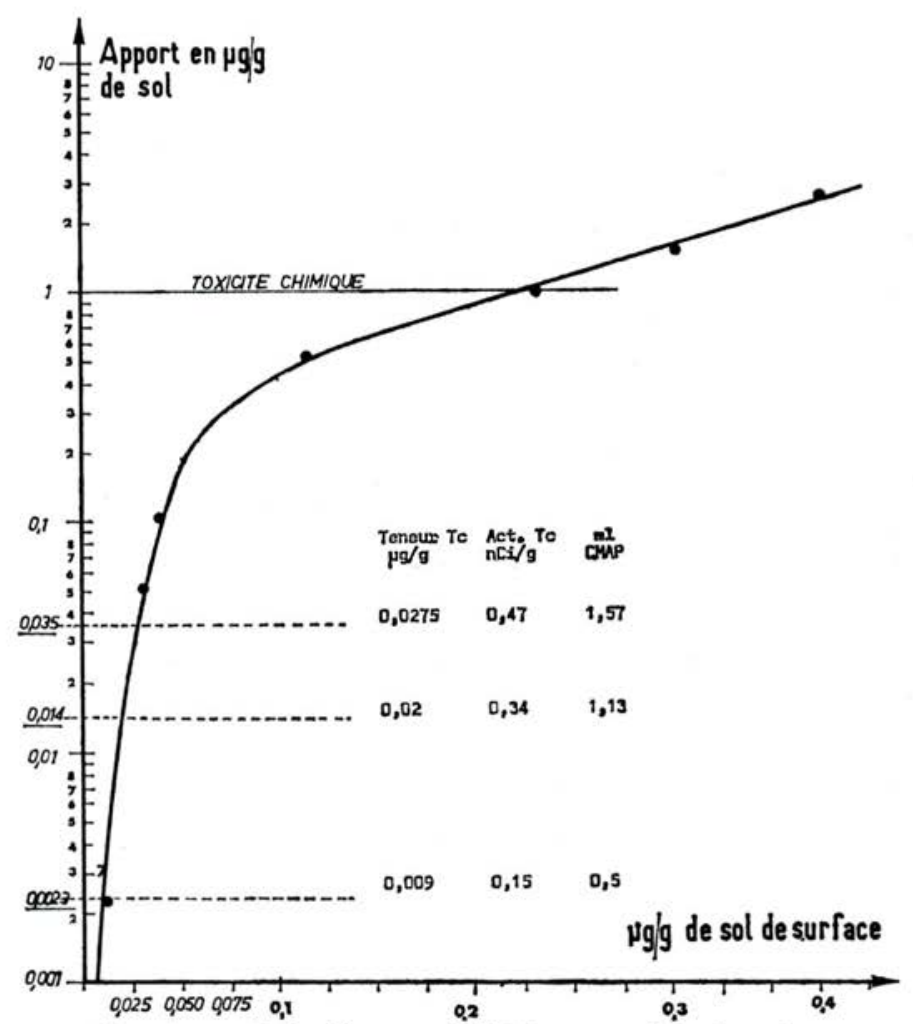

FIG. 9. - Technétium assimilable en surface du sol en fonction de l'apport initial après une culture de salade.

Irrigation cumulée pour une culture de salade équivalente à $240 \mathrm{~mm}$ de hauteur d'eau

Le dépôt de technétium est de $0,42 \mu \mathrm{g} / \mathrm{cm}^{2}$; la teneur du sol dans 1'hypothèse d'un dépôt uniforme sur $20 \mathrm{~cm}$ est de $0,014 \mu \mathrm{g} / \mathrm{g}$ de sol; la lecture de la courbe expérimentale permet d'évaluer le dépôt superficiel à $0,052 \mu \mathrm{g} / \mathrm{g}$ soit $0,89 \mathrm{nCi} / \mathrm{g}$ équivalent à $2,96 \mathrm{ml}$ d'eau à la CMAP. On observe donc encore une concentration en surface.

Irrigation moyenne annuelle de $600 \mathrm{~mm}$ de hauteur d'eau

Le dépôt résultant de cette irrigation est de $1,05 \mu \mathrm{g} / \mathrm{cm}^{2}$ soit $0,035 \mu \mathrm{g} / \mathrm{g}$ de sol sur $20 \mathrm{~cm}$ de profondeur; après culture de salade, le dépôt résiduel 
à la surface du sol est de $0,08 \mu \mathrm{g} / \mathrm{g}$ soit $1,37 \mathrm{nCi} / \mathrm{g}$ équivalent à $4,56 \mathrm{ml}$ d'eau à la CMAP. Dans ce cas, comme précédemment, il y a accumulation à la surface du sol.

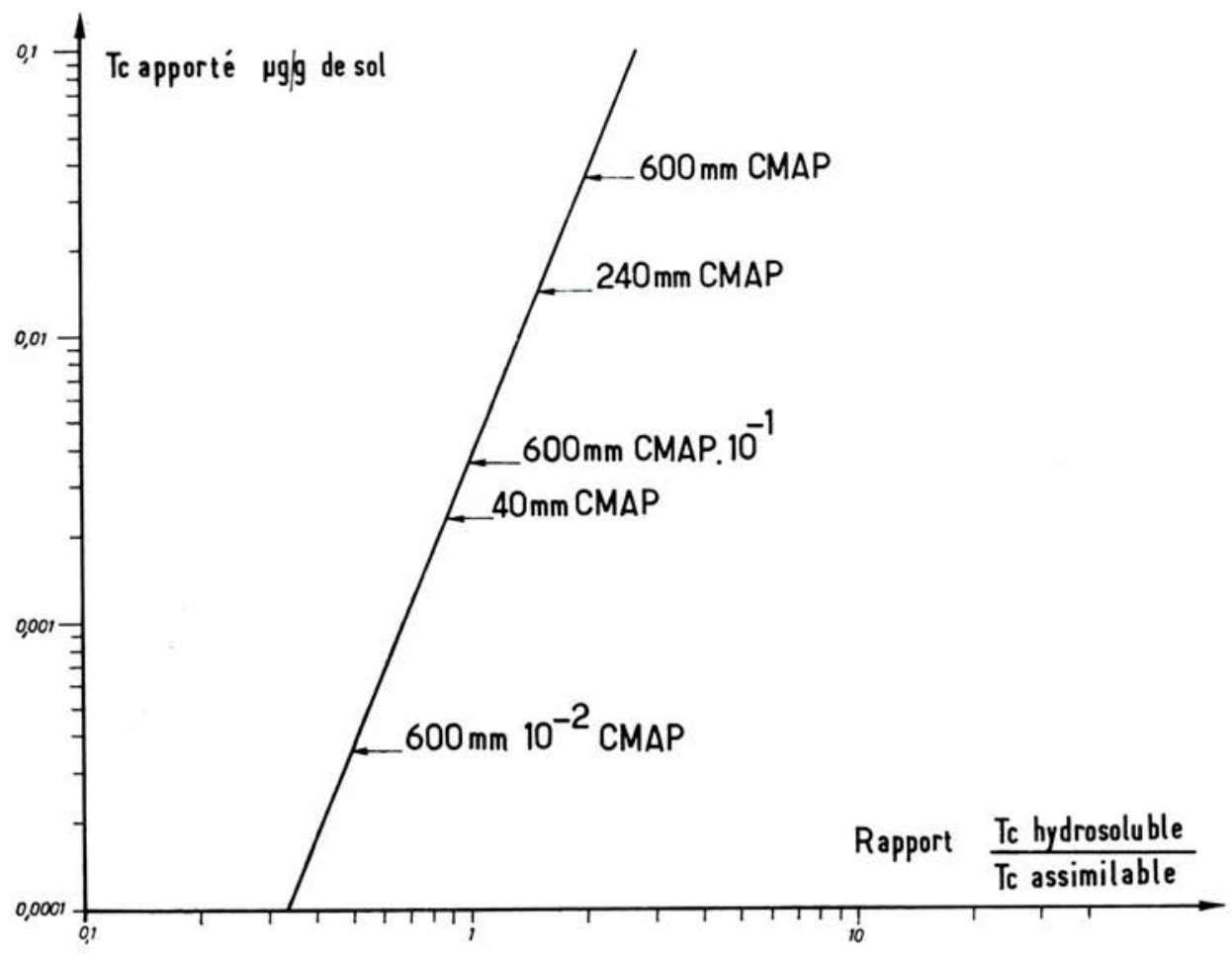

FIG. 10. - Évolution du rapport technétium hydrosoluble/technétium assimilable en fonction de l'apport initial.

L'utilisation de la courbe expérimentale de la figure 7 nous montre qu'inévitablement tout apport de technétium par les eaux d'irrigation, même en quantités très faibles, se traduit par une accumulation à la surface du sol. Cette observation corrobore les résultats de l'élution qui indiquaient que, pour de très faibles quantités de technétium apportées au sol, la perte par lessivage est quasiment nulle.

\subsection{Formes PHysico-ChimiQues dU teChNÉTIUM A LA SURFACE DU SOL}

Si l'accumulation du technétium à la surface du sol permet d'effectuer une première prévision concernant l'augmentation progressive de la teneur en technétium des sols irrigués, il faut cependant déterminer la forme physicochimique de cet isotope pour évaluer la disponibilité pour les végétaux, ceci 
surtout dans la zone de labour. C'est pourquoi, sur les échantillons prélevés précédemment nous avons extrait, d'une part, le technétium soluble à l'eau ( fig. 8) et le technétium assimilable par le réactif de Grigg ( fig. 9), d'autre part. L'observation des résultats nous montre que l'allure générale des courbes, représentant les deux formes de technétium, est similaire à celle de la figure 7 . Lorsque la quantité de technétium apportée au sol augmente, la teneur à l'état hydrosoluble croît rapidement. Cependant, pour les trois cas d'irrigation retenus, nous obtenons, à partir de la courbe expérimentale, les valeurs suivantes :

- $40 \mathrm{~mm}$ de hauteur d'eau : 0,008 $\mu \mathrm{g} / \mathrm{g}$;

- $240 \mathrm{~mm}$ de hauteur d'eau : $0,030 \mu \mathrm{g} / \mathrm{g}$;

- $600 \mathrm{~mm}$ de hauteur d'eau : $0,055 \mu \mathrm{g} / \mathrm{g}$.

Pour les faibles valeurs de dépôt il n'y a donc pas augmentation proportionnelle entre l'apport et la teneur résiduelle du technétium hydrosoluble. Le végétal doit certainement absorber cette forme par voie racinaire; dans cette hypothèse, il faut alors admettre que l'absorption racinaire n'est pas proportionnelle au dépôt; nous verrons, dans une étude ultérieure, ce qu'il en est exactement de l'assimilation du technétium par les végétaux dans le cas des faibles dépôts [4]. En ce qui concerne la fraction de technétium extractible au réactif de Grigg, nous observons sur la figure 9 que cette forme mérite surtout d'être étudiée pour les dépôts faibles; en effet, la capacité de fixation du technétium sous cette forme parait être limitée.

A la vue de ces résultats, il est donc intéressant de tracer la courbe qui donne l'évolution du rapport technétium hydrosoluble/technétium assimilable en fonction du dépôt au sol ( fig. 10). Nous obtenons une droite qui met en évidence une progression géométrique du rapport (progression 1/2); une progression géométrique du dépôt (progression $10^{-1}$ exprimée en CMAP). Ainsi, pour des irrigations annuelles de $600 \mathrm{~mm}$ à diverses concentrations, nous pouvons aisément estimer le rapport entre les deux formes physico-chimiques :

\begin{tabular}{|c|c|}
\hline CMAP & $\begin{array}{c}\text { Rapport } \\
\text { hydrosoluble/assimilable }\end{array}$ \\
\hline $1 \ldots \ldots \ldots$ & 2 \\
\hline $10^{-1} \ldots \ldots \ldots$ & 1 \\
\hline $10^{-2} \ldots \ldots \ldots$ & $0,5 \quad x=600 \mathrm{~mm} \mathrm{CMAP}$ \\
\hline $10^{-3} \ldots \ldots \ldots$ & $0,25 \quad=5,26 \cdot 10^{-2} \mu \mathrm{g} / \mathrm{g}$ de sol $)$ \\
\hline $10^{-4} \ldots \ldots \ldots$ & 0,125 \\
\hline
\end{tabular}

Le rapport entre les deux formes physico-chimiques $(y)$ est lié à la concentration $\left(x^{\prime}\right)$ exprimée en CMAP par la relation :

$$
y=2 x^{\prime 0,30} \text {. }
$$




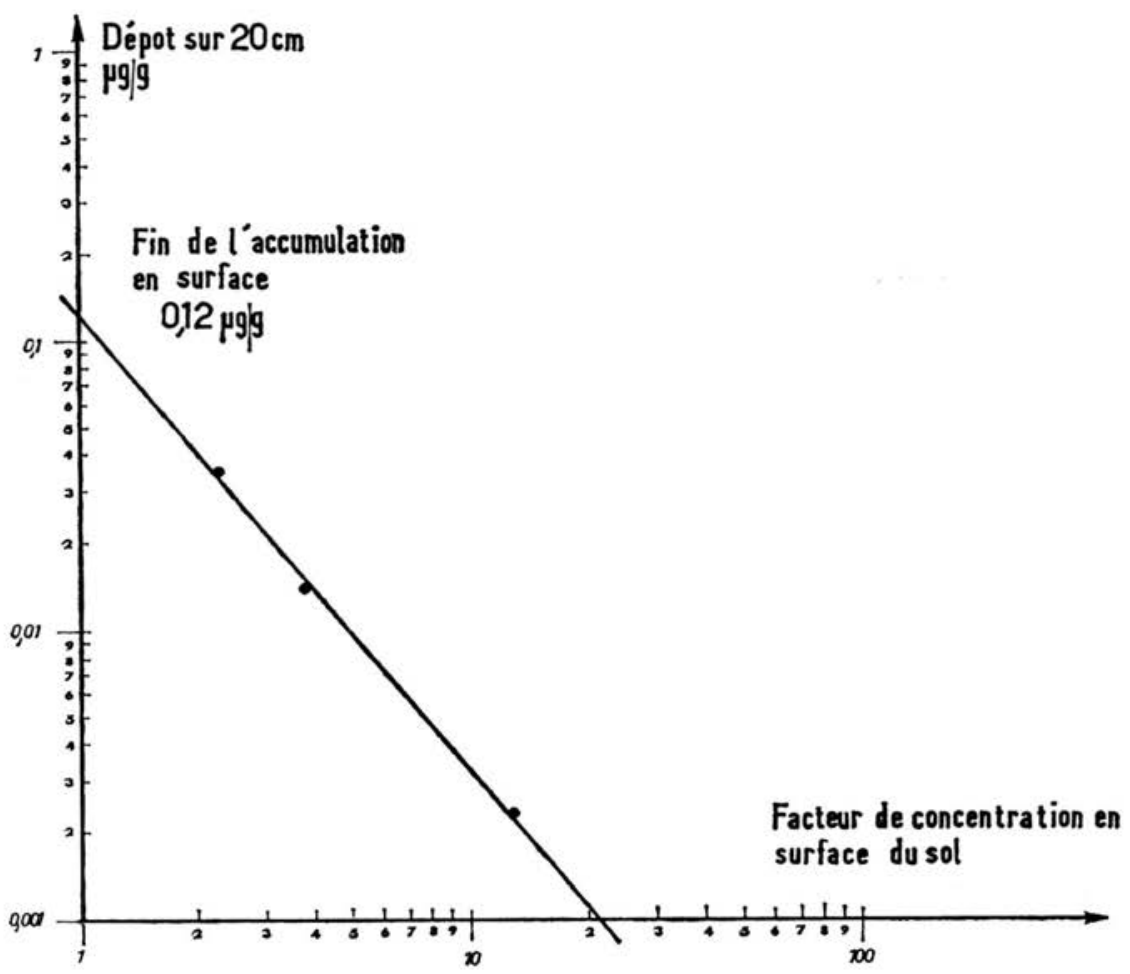

FIG. 11. - Détermination de la limite de concentration à la surface du sol $(\mu \mathrm{g} / \mathrm{g})$.

C'est donc la rétention de technétium à l'état assimilable qui empêche le lessivage de cet isotope pour des apports faibles (CMAP ou inférieure à la CMAP).

La comparaison entre la somme totale de technétium retenu à la surface du sol et le fractionnement en formes physico-chimiques montre également que, pour les très faibles quantités de technétium apportées au sol, il subsiste une fraction de technétium non extractible. Cette quantité, pour le sol brun calcaire, est comprise entre 0,001 et $0,002 \mu \mathrm{g} / \mathrm{g}$, ce qui correspond à peu près à l'équivalent déposé par une irrigation de $40 \mathrm{~mm}$ de hauteur d'eau à la CMAP en supposant la répartition uniforme sur $20 \mathrm{~cm}$. Si l'on admet que cette forme de technétium est peu disponible pour les végétaux, l'absorption racinaire doit être plus faible pour des dépôts inférieurs à $0,001 \mu \mathrm{g} / \mathrm{g}$. Nous verrons dans une communication ultérieure sur l'absorption racinaire ce qu'il en est exactement de l'exportation du technétium par les végétaux pour des dépôts très faibles [4].

A partir de ces données, nous allons à présent évaluer les concentrations maximales à la surface du sol pour des irrigations avec des eaux à la CMAP ou inférieures à la CMAP. 


\subsection{ESTIMATION DU TECHNÉTIUM A LA SURFACE DU SOL POUR DES IRRIGATIONS AVEC DE L'EAU A LA CMAP OU INFÉRIEURE A LA CMAP}

Les observations précédentes, tirées des valeurs expérimentales, confirment que le technétium s'accumule à la surface du sol et que ce mécanisme est dû, semble-t-il, à la rétention d'une forme non extractible $(0,001$ à $0,002 \mu \mathrm{g} / \mathrm{g}$ de sol) et à l'existence d'une forme assimilable variable en fonction de l'apport.

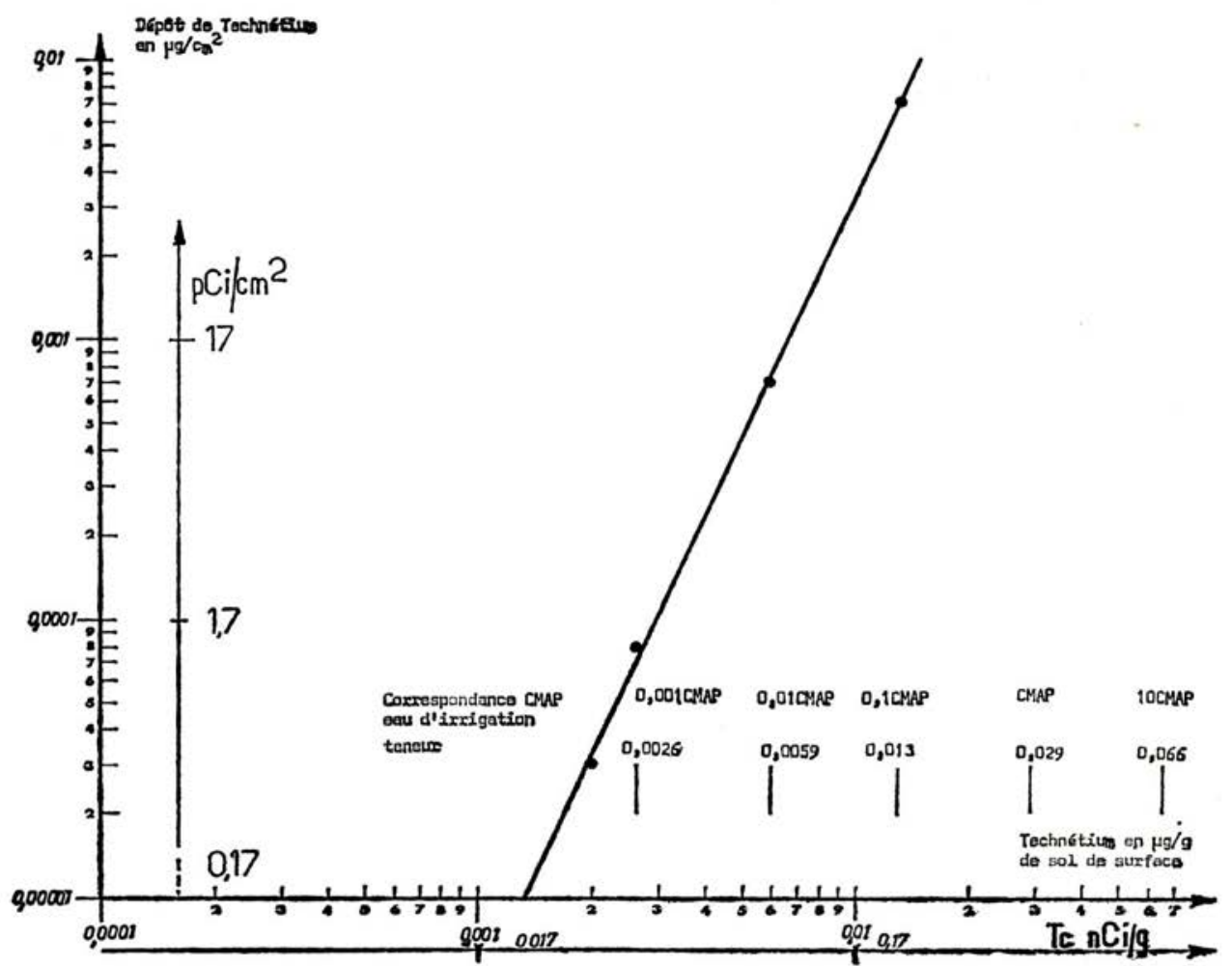

Fig. 12. - Concentration du technétium en surface du sol pour une irrigation de $40 \mathrm{~mm}$ à diverses CMAP.

L'évaluation de la concentration finale après culture peut se faire à partir des résultats expérimentaux. Le facteur de concentration (rapport entre la teneur initiale et la quantité résiduelle) pour les diverses expériences donne une droite ( $f$ fig. 11). On peut admettre que nous atteignons la limite supérieure de l'accumulation lorsque le facteur de concentration est équivalent à 1. Cette limite supérieure pour le sol brun calcaire est de $0,12 \mu \mathrm{g} / \mathrm{g}$. Ceci correspond à un dépôt de $3,6 \mu \mathrm{g} / \mathrm{cm}^{2}$ soit $205 \mathrm{ml}$ d'eau d'irrigation à la CMAP. Cette hauteur d'eau peut être atteinte en 3 ou 4 ans. Pour cette valeur limite, le rapport technétium hydrosoluble/technétium assimilable est de 1,4. 


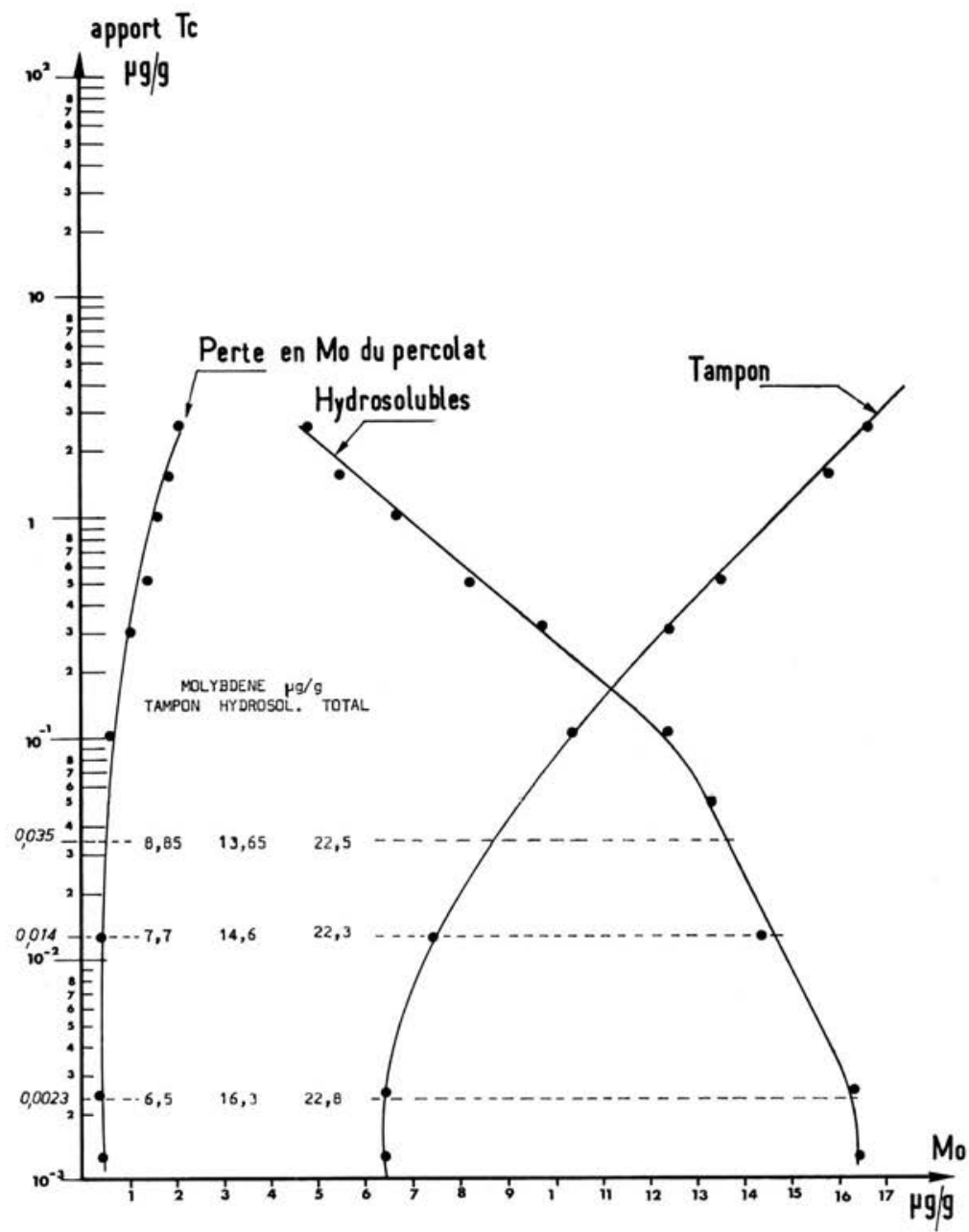

FIG. 13. - Formes du molybdène dans le sol en fonction de l'apport en technétium après une culture de salade.

La teneur en surface de $0,12 \mu \mathrm{g} / \mathrm{g}$ correspond à un apport initial de $0,15 \mu \mathrm{g} / \mathrm{g}$ selon la figure 7 , soit un dépôt de $4,5 \mu \mathrm{g} / \mathrm{cm}^{2}$. Dans ces conditions, le dépôt initial/ha sur $20 \mathrm{~cm}$ de profondeur est de $450 \mathrm{~g} / \mathrm{ha}$; l'exportation par une culture de salade (facteur de concentration 30) [4] de $180 \mathrm{~g} / \mathrm{ha}$; la valeur moyenne résiduelle sur $20 \mathrm{~cm}$ sera de $0,08 \mu \mathrm{g} / \mathrm{g}$.

La figure 12, dérivée de la figure 7 permet d'évaluer le dépôt en surface du sol pour des irrigations uniques de $40 \mathrm{~mm}$ de hauteur d'eau contenant diverses teneurs de technétium. Nous obtenons par l'intermédiaire de cette 
courbe deux progressions géométriques : celle de l'apport, exprimée en puissance de 10 par rapport à la CMAP, celle de la concentration à la surface du sol de raison 2,2 à partir d'une valeur $\mathrm{A}$ (spécifique au sol). La concentration à la surface du sol $(y)$, pour des irrigations avec des eaux inférieures à la CMAP, est liée directement à la concentration de l'eau en technétium $(x)$ exprimée en CMAP par la relation :

$$
y=\mathrm{A} \times 2,2^{\log x-1} .
$$

Dans le cas présent, $\mathrm{A}=0,066 \mu \mathrm{g}$ pour une concentration de $10 \mathrm{CMAP}$. L'équation générale pour la teneur en technétium du sol $\left(y^{\prime}\right)$ par rapport à la concentration dans l'eau $\left(x^{\prime}\right)$ s'écrit :

$$
y^{\prime}=\frac{x^{\prime 0,35}}{33,3} .
$$

Cette relation est du même type que celle qui régit l'équilibre entre les formes physicochimiques.

L'ensemble de ces relations, obtenues à partir des valeurs expérimentales, confirme que, même pour des concentrations très faibles de technétium à partir des eaux d'irrigation, on assiste à une accumulation à la surface du sol et que, par ailleurs, cette accumulation peut croître jusqu'à une valeur limite de $0,12 \mu \mathrm{g} / \mathrm{g}$ ce qui correspond à un dépôt de $4,5 \mu \mathrm{g} / \mathrm{cm}^{2}$ dans le cas de notre sol brun calcaire. Étant donné que les relations ci-dessus tiennent compte de la variable $\log x$, c'est-à-dire de la concentration du technétium, il y a tout lieu de penser $q u ' e n$ ce qui concerne le coefficient de partage entre l'eau d'irrigation et le sol, c'est avec un coefficient de partage pondéral, qui répond à tout moment à la loi d'action de masse, qu'il faudra travailler. Ces relations montrent par ailleurs que malgré les prélèvements du végétal, il y a néanmoins accumulation dans le sol.

\section{COMPORTEMENT DU MOLYBDÈNE}

\section{A LA SURFACE DU SOL}

Le molybdène présent dans les échantillons prélevés en surface a été extrait par les mêmes réactifs que le technétium ( fig. 13). Cette figure montre que la perte du molybdène par percolation est sensible à partir d'un dépôt de $0,1 \mu \mathrm{g} / \mathrm{g}$ de sol de technétium (rapport $\mathrm{Mo} / \mathrm{Tc} \simeq 200$ ). Il se produit parallèlement à ce phénomène de lessivage une variation sensible dans les formes physico-chimiques extraites; en effet, un apport de technétium supérieur à $10^{-2} \mu \mathrm{g} / \mathrm{g}$ diminue le molybdène hydrosoluble qui, de ce fait, est éliminé par percolation; parallèlement, on observe une augmentation des formes dites assimilables. L'apport de technétium au niveau du sol favorise, d'une part, la fixation du molybdène sous forme assimilable et, d'autre part, il augmente le lessivage dans le profil. 
La relation qui exprime le rapport technétium hydrosoluble/technétium assimilable par rapport au dépôt montre que, très rapidement, les formes hydrosolubles sont prépondérantes; celles-ci se mettent en équilibre avec le molybdène tout en changeant la répartition de ce dernier. Au niveau du sol, le technétium peut donc remplacer partiellement le molybdène au niveau des sites de fixation; sa plus grande solubilité aboutit, d'une part, à l'élimination des formes hydrosolubles du molybdène, d'autre part, à sa fixation à l'état assimilable. Cette observation corrobore l'existence des seuils pour le rapport $\mathrm{Mo} / \mathrm{Tc}$ au niveau de l'élution. De ce fait, il n'est pas impensable que, sur le plan de l'absorption racinaire, le technétium puisse remplacer partiellement le molybdène, notamment lorsque cet oligo-élément est peu abondant et qu'il y a carence vis-à-vis des végétaux.

\section{CONCLUSIONS}

Cette étude expérimentale, pour laquelle nous n'avons retenu que l'évolution du technétium au niveau du sol, nous conduit à formuler un certain nombre de remarques qui sont essentiellement valables pour le sol brun calcaire à réaction alcaline :

- l'apport conjoint de technétium et de molybdène entraîne une interaction du comportement de ces isotopes dans le sol; l'élution de ces radionucléides est fonction du rapport $\mathrm{Mo} / \mathrm{Tc}$ présent dans le sol et, dans le cas de notre étude expérimentale, il apparaît deux seuils pour lesquels il y a une différence notable de lessivage;

- la formulation mathématique du lessivage de ces éléments aboutit à la conclusion que le technétium n'est pas lessivé lorsque les apports sont faibles; le corollaire de cette observation se traduit par une accumulation au niveau du sol et plus spécialement en surface;

- l'examen des formes physico-chimiques résiduelles dans le sol après une culture de salade met en évidence l'existence de trois formes : une forme non extractible fortement liée au sol qui représente 1 à $2 \cdot 10^{-3} \mu \mathrm{g} / \mathrm{g}$ de sol, une forme assimilable, une forme hydrosoluble. Le rapport entre ces deux formes est fonction du dépôt et c'est ce rapport qui conditionne le lessivage. Ce lessivage intervient lorsque la valeur résiduelle est supérieure à $0,12 \mu \mathrm{g} / \mathrm{g}$ de sol dans notre cas;

- les résultats concernant les formes physico-chimiques du molybdène résiduel confirment l'élimination du molybdène hydrosoluble pour des apports élevés de technétium et le déplacement de l'équilibre des formes physicochimiques, ce qui pourrait avoir pour conséquence une différence d'absorption racinaire.

Sur le plan du cycle de cet isotope dans l'environnement et de la radioprotection, cette étude nous conduit, par ailleurs, à formuler les remarques suivantes :

- le technétium est à considérer comme un isotope pondéral avec toutes les conséquences qui en découlent (loi d'action de masse, synergie et antagonisme vis-à-vis d'autres isotopes stables, etc.);

voL. $14-\mathrm{N}^{\circ} 4$ 
- l'accumulation au niveau du sol se produit, même pour des quantités très faibles d'apport, l'accumulation limite dans le sol peut être obtenue par des irrigations du sol avec des eaux à la CMAP ;

- étant donné les informations sur la toxicité chimique de cet élément vis-à-vis des végétaux, nous sommes en droit de nous demander s'il n'y a pas toxicité au niveau du sol lorsque nous atteignons la limite maximale d'accumulation, cette étude devra donc être entreprise;

- par ailleurs, il apparaît que le cycle du technétium est dépendant en grande partie du rapport $\mathrm{Mo} / \mathrm{Tc}$ dans le sol; il est donc important de fixer, d'une part, les seuils d'élution et, d'autre part, les seuils d'accumulation dans le cas des principaux types de sols;

- les possibilités d'élution de cet isotope laissent entrevoir que la nappe phréatique est le milieu récepteur par excellence à la fois dans le cadre des échanges fleuve-nappe et dans le cadre des alimentations des nappes par ruissellement et lessivage. Les équations calculées pour le rapport entre les formes physicochimiques du technétium et sa vitesse d'accumulation au sol permettent, dans ces cas, de prévoir lorsque ce compartiment nappe sera touché par le cycle.

Sans préjuger $a$ priori des problèmes posés par les relations sol-plante que nous présentons dans un autre rapport [4], il apparaît que l'évolution du technétium dans le sol mérite, à elle seule, un certain nombre de confirmations, en particulier l'évaluation de la toxicité et l'influence des paramètres : types de sol, qualité d'eau d'irrigation, pluviométrie. Néanmoins, dans le cas de notre expérimentation, le cycle du technétium 99 se pose sous un triple aspect : radioactif, pondéral et toxique chimique.

\section{BIBLIOGRAPHIE}

[1] LANDA E. R. The behaviour of technetium 99 in soils and plants. Thèse, Minnesota University, Minneapolis, 1975.

[2] Gast R. G. The behaviour of technetium 99 in soils and plants, progress report, 19751976, COO-2447-4, 1977.

[3] Saas A., Quinault J. M., Denardi J. L., Colle C. Estimation du cycle molybdène dans divers types de sols dans l'environnement des sites électronucléaires. Classification des sols. Évaluation des formes physico-chimiques (communication personnelle).

[4] SaAs A., Grauby A., Denardi J. L. Absorption racinaire du technétium. Évaluation des conséquences sur le plan sanitaire et de la physiologie végétale (à paraître).

[5] Trll S. E., Hoffman F. O., Dunning D. E. Jr. A new look at ${ }^{99}$ Tc releases to the atmosphere. Health Phys., 1979, 36, 21-30.

[6] Wildung R. E., Garland T. R., Cataldo D. A. Accumulation of technetium by plants. Health Phys., 1977, 32, 314-317. 